\title{
Generation of photons with all-optically-reconfigurable entanglement in integrated nonlinear waveguides
}

\author{
James G. Titchener, Alexander S. Solntsev, and Andrey A. Sukhorukov \\ Nonlinear Physics Centre, Research School of Physics and Engineering, Australian National University, Canberra ACT 2601, Australia
}

(Received 3 November 2014; published 11 September 2015)

\begin{abstract}
We predict that all-optically-reconfigurable generation of photon pairs with tailored spatial entanglement can be realized via spontaneous parametric down conversion in integrated nonlinear coupled waveguides. The required elements of the output quantum wave function are directly mapped from the amplitudes and phases of the classical laser pump inputs in each waveguide. This is achieved through special nonuniform domain poling, which locally inverts the sign of quadratic nonlinear susceptibility and accordingly shapes the interference of biphoton quantum states generated along the waveguides. We demonstrate a device configuration for the generation of any linear combination of two-photon Bell states.
\end{abstract}

DOI: 10.1103/PhysRevA.92.033819

PACS number(s): 42.65.Wi, 42.65.Lm, 42.50.-p, 42.82.Et

\section{INTRODUCTION}

Entanglement is a key characteristic of quantum mechanics, and as shown by Bell [1], is responsible for quantum theories' violation of the classical principle of local relativistic causality. As well as being of fundamental scientific interest, quantum entanglement can enable powerful new technologies such as quantum computing [2], quantum communication [3], and quantum enhanced measurement [4]. However, the highly advantageous nonclassical properties of these technologies will require a flexible interface between the classical and quantum worlds. Indeed, the development of such an interface is an area of active research [5-8], the dominant method being spontaneous parametric down conversion (SPDC), where a classical laser interacts with a $\chi^{(2)}$ nonlinear medium to produce entangled photons $[5,6,8]$.

Photons are an ideal platform for creating and manipulating quantum information due to the low noise from the environment and ease of transmission. A qubit encoded into a photon can be easily sent between different photonic elements along an optical fiber, in analogy to the transmission of classical bits along electrical wires [2]. Furthermore, logic operations can be performed on entangled photons by exploiting the nonlinearity inherent in quantum measurement, or by using the interaction between multiple light fields via a nonlinear medium [2,9]. However, in order for these properties to be fully utilized, it must first be possible to quickly map information onto the quantum state of entangled photons. Therefore, an entangled photon source with fast all-optical reconfigurability of the output wave function would be an important step towards realizing new quantum technologies.

The practicality of any of these technologies will be determined by their scalability and reliability; this suggests the use of quantum photonic circuits will be essential. Integrating optical elements onto a single chip reduces the systems contact with the environment, preserving the fidelity of the quantum entanglement by reducing phase fluctuations and other sources of noise [10]. Furthermore, integrated devices are compact and stable, so they can be combined to build complex quantum circuits that would be impossibly large using traditional bulk optics. Hence, a practical source of entanglement will require the generation and control of entangled photons to be integrated within a single photonic chip.
The latest experimental developments [6,8,11-13] suggest that the goal of a fully integrated and optically reconfigurable source of entangled photons is achievable, but there is still some way to go. For instance, Refs. $[6,11]$ show that entangled photons could be created on-chip, while $[12,13]$ demonstrate that the reconfigurable manipulation of entangled photons in quantum photonic circuits is possible. Indeed, the on-chip generation of photon pairs and their interference using tunable phase shifters was demonstrated in Refs. [8,13], although all-optical reconfigurability as well as the ability to generate a full set of Bell states and their superpositions are yet to be achieved.

In this work, we predict that a photonic chip consisting of an array of coupled nonlinear waveguides [14-16] can be designed for all-optically-controlled generation of any set of path-entangled biphoton states. This device is particularly elegant because the output quantum wave function is directly mapped from the amplitudes and phases of the classical laser inputs. Hence, the device can be reconfigured in real time by varying classical inputs, providing a flexible interface between classical and quantum information.

This paper is organized as follows. In Sec. II, we introduce the nonlinear waveguide array (WGA), a device that can produce entangled photon pairs with correlations between photons in different waveguides. In Sec. III, we show how to design $\chi^{(2)}$ poling structures in the WGA to allow the production of nearly any desired two-photon state when pumping just a single waveguide. As a specific example, we show how to structure the poling so that pumping each waveguide in an array of four coupled waveguides produces a different Bell state. Then, in Sec. IV we investigate the limits of using $\chi^{(2)}$ poling patterns to produce any arbitrary two-photon wave function, showing that not all wave functions can be produced in a homogeneous WGA. In Sec. V, we show that these limits can be overcome by altering the widths of some waveguides in the WGA in order to break the symmetry of the device. In Sec. VI, we show how the device can be characterized quickly using classical frequency difference generation. Finally, in Sec. VII, we numerically model the effect of realistic fabrication errors on the performance of the device. We present conclusions in Sec. VIII. 
(a)

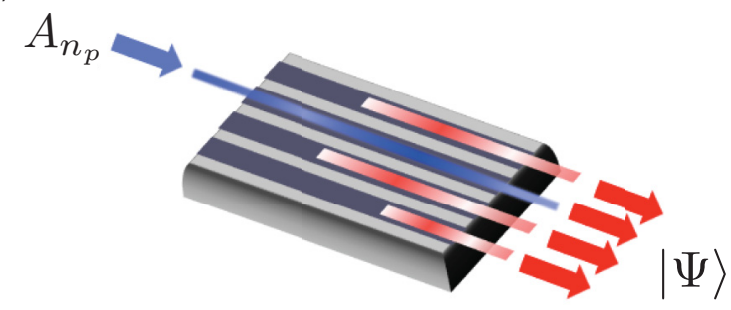

(b)

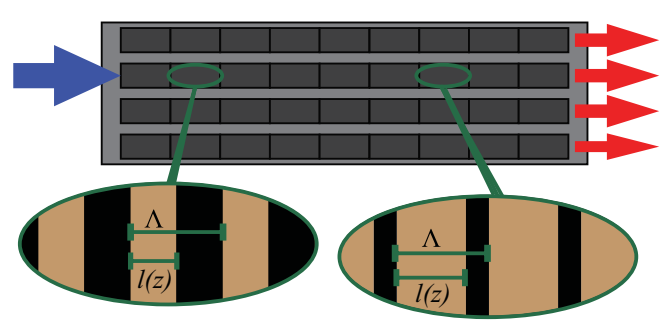

FIG. 1. (Color online) (a) Diagram of quadratically nonlinear waveguide array. A pump laser field $A_{n_{p}}$ produces path-entangled two-photon state $\Psi$ via SPDC. (b) Poling pattern for wave-function control: each waveguide is divided into a number of segments, with a different duty cycle (ratio of "up" to "down" poling) in each segment.

\section{DEVICE DESIGN AND MODEL EQUATIONS}

We illustrate the device concept in Fig. 1(a). Laser driving one of the waveguides in the nonlinear waveguide array will generate entangled photon pairs in the driven waveguide via SPDC. The waveguides are coupled such that the biphotons (entangled photon pairs) can tunnel between neighboring waveguides, but the pumping laser is confined to one waveguide. This is an example of a driven quantum walk [17] of pairs of entangled particles. It has been shown that the interference between the probability amplitudes of different biphoton paths can lead to highly nonclassical states at the output of the device $[14,15]$. However, it remained an open question as to whether the WGA could be tuned to produce custom and reconfigurable quantum states. We demonstrate that this can be achieved through specially designed domain poling, as illustrated in Fig. 1(b).

To model SPDC in the waveguide array we work in the weak pump regime, neglecting all terms in the state of the downconverted photons corresponding to the creation of multiple photon pairs. Hence, we assume that SPDC produces a twophoton quantum state, which can be described as

$$
\begin{aligned}
|\Psi(z)\rangle= & \iint d \omega_{s} d \omega_{i} \\
& \times \sum_{n_{s} n_{i}}^{N} \Psi_{n_{s} n_{i}}\left(z, \omega_{s}, \omega_{i}\right) a_{n_{s}}^{\dagger}\left(\omega_{s}\right) a_{n_{i}}^{\dagger}\left(\omega_{i}\right)|0\rangle_{s}|0\rangle_{i},
\end{aligned}
$$

where $a_{n}^{\dagger}(\omega)$ is the creation operator for a photon of frequency $\omega$ in waveguide number $n \in[1,2, \ldots, N]$ and $\Psi_{n_{s} n_{i}}\left(z, \omega_{s}, \omega_{i}\right)$ is the biphoton wave function which depends on both the frequency and position of the signal (subscript $s$ ) and idler $(i)$ photons in the WGA.

We consider filtering the frequency range of the output biphoton state to a narrow band such that the signal and idler are nearly degenerate. However, mathematically we must still treat the photons as distinguishable because true degeneracy occurs only at an infinitesimal point on the spectrum. Restricting both the integrals over signal and idler frequencies to a single, sufficiently narrow frequency range, the two-photon state is described by a frequency-independent wave function

$$
\Psi_{n_{s} n_{i}}\left(z, \omega_{s}, \omega_{i}\right) \approx \Psi_{n_{s} n_{i}}(z) .
$$

When pumping the WGA with a laser of frequency $\omega_{p}=$ $\omega_{s}+\omega_{i}$, the biphoton wave function obeys the differential equation $[15,18]$

$$
\begin{aligned}
i \frac{\partial \Psi_{n_{s}, n_{i}}(z)}{\partial z}= & i \sum_{n_{p}=1}^{N} A_{n_{p}} d_{n_{p}}(z) e^{i \Delta \beta^{(0)} z} \delta_{n_{s}, n_{p}} \delta_{n_{i}, n_{p}} \\
& -C\left[\Psi_{n_{s}, n_{i}+1}+\Psi_{n_{s}, n_{i}-1}+\Psi_{n_{s}+1, n_{i}}+\Psi_{n_{s}-1, n_{i}}\right] .
\end{aligned}
$$

Here, the first term on the right is the generation of new biphotons via SPDC, with classical laser driving amplitude $A_{n_{p}}$ and second-order nonlinear coefficient $d_{n_{p}}(z)$ in waveguide number $n_{p}$, while the phase mismatch is denoted as $\Delta \beta^{(0)}$. The last term on the right describes the evanescent coupling of signal and idler photons between neighboring waveguides with the coupling rate given by $C$. Due to the symmetry in Eq. (3), for the initial vacuum state $\Psi_{n_{s}, n_{i}}(0)=0$, the biphoton wave function is symmetric, $\Psi_{n_{s}, n_{i}}(z)=\Psi_{n_{i}, n_{s}}(z)$, at all $z$.

It is important to note that the propagation of biphotons in a nonlinear waveguide array is essentially linear. So, if driving the waveguide $n_{p}$ with unity pump amplitude produces the quantum output state $\left|\psi_{n_{p}}\right\rangle$, then driving multiple waveguides produces the state $|\Psi\rangle=\sum_{n_{p}=1}^{N} A_{n_{p}}\left|\psi_{n_{p}}\right\rangle$. Therefore, by varying the $N$ classical laser inputs $A_{n_{p}}$, we can reconfigure the device in real time to produce any state in an $N$-dimensional quantum space. However, the total quantum output space will have $N(N+1) / 2$ degrees of freedom, namely, the complex wave-function amplitudes of pairs of photons occupying any two of $N$ waveguides. Thus, by varying the $N$ lasers driving the device, only a subspace of the full set of possible output wave functions can be produced. So the ability to all-optically vary the output wave function is limited by the fact that only $N$ of the $N(N+1) / 2$ degrees of freedom can be accessed.

We address this problem, showing that the output space can be flexibly controlled by introducing special domain poling patterns in the WGA [Fig. 1(b)]. Adjusting the domain poling pattern allows us to choose the exact form of the $N$ dimensional subspace that is spanned by varying the classical laser inputs to the device. This is achieved by optimizing the poling in each waveguide to produce a specific "basis state" $\left|\psi_{n_{p}}\right\rangle$, when it is pumped individually. Then, pumping all waveguides simultaneously will allow the creation of any linear combination of the "basis states."

\section{POLING STRUCTURES}

The typical use of domain poling is to achieve quasiphase matching (QPM) for $\chi^{(2)}$ nonlinear processes [19]. QPM involves periodically inverting the orientation of the ferroelectric dipole moment in the nonlinear medium; this 
corresponds to altering the sign of the second-order nonlinearity in the medium. The technique has also been used to shape the wavefront of down-converted photons in bulk nonlinear crystals $[5,6,20,21]$. We utilize domain poling within the waveguide array structure to alter the sign of the nonlinear coefficient $d_{n_{p}}(z)$ as a function of waveguide number $n_{p}$ and propagation length $z$. This effectively inverts the quantum phase of biphotons generated at different points along the array, enabling controlled interference between different biphoton paths.

We begin by discussing the limits on the size of ferroelectric domains, and how the limits are accounted for in our design. With established fabrication techniques the minimum length (or width) of a ferroelectric domain that can be fabricated is around $5 \mu \mathrm{m}$ [22], while newer techniques promise smaller domain sizes [23]. In contrast, the typical separation distance between waveguide cores for the type of coupled WGA we consider is greater than $10 \mu \mathrm{m}$. Therefore, it is entirely possible to envisage a device comprised of coupled waveguides with a different poling pattern in each waveguide. In order to shape the biphoton wave function, the efficiency of SPDC is varied along the length of each waveguide by changing the duty cycle of the poling pattern (the ratio of "up" to "down" poling) between $0 \%$ and $50 \%$. This would generally result in domains that are too small to fabricate since having an arbitrarily small duty cycle would require arbitrarily small domain sizes. In Sec. VII, we show that doubling the period of the poling pattern allows our method to work using only duty cycles between $25 \%$ and $50 \%$. For lithium niobate, this results in a minimum required domain size of around $10 \mu \mathrm{m}$, which should be achievable with established fabrication procedures.

As in QPM we alter the nonlinear coefficient to create poling structure with periodicity that tends to cancel out the phase mismatch of the SPDC process. However, as mentioned above, we also modulate the local efficiencies of SPDC by varying the duty cycle of the periodic poling structure. This controlled variation of SPDC efficiency allows tailoring of the output biphoton wave function. Note that varying the duty cycle is not the only way to achieve this variation: the poling could be structured in many different ways to achieve the desired variation of SPDC efficiency. Hence, there is great flexibility in the design process to accommodate fabrication limitations. This leads us to formulate the problem more generally in terms of the "aggregate nonlinearity," the average efficiency of the down-conversion process over one (or a small number of) coherence lengths of SPDC, $L_{c}=2 \pi / \Delta \beta^{(0)}$.

Using the aggregate nonlinearity makes it possible to find a general solution for the poling pattern required to produce any arbitrary biphoton wave function. This general solution amounts to solving Eq. (3) for $\Psi_{n_{s} n_{i}}(L)$ and then inverting the result to express the poling structure $d_{n_{p}}(z)$ in terms of $\Psi_{n_{s} n_{i}}(L)$. The role of the aggregate nonlinearity is to mediate between the discrete up-down poling structure and the continuous valued biphoton wave function.

The solution to Eq. (3) is found in terms of the supermodes of the coupled waveguide array. These are the eigenfunctions of the interwaveguide coupling operator and are denoted $f_{k_{s}, k_{i}}(z)$, where $k_{s}$ and $k_{i}$ are the discrete wave vectors of the signal and idler within the array. Solving Eq. (3) gives the following solution for the biphoton wave function in terms of amplitude of each supermode at the end of the array:

$$
\begin{aligned}
f_{k_{s}, k_{i}}(L)= & e^{i \beta_{k_{s} k_{i}} L} \sum_{n_{p}=1}^{N} A_{n_{p}} \sin \left(\frac{\pi k_{i} n_{p}}{N+1}\right) \sin \left(\frac{\pi k_{s} n_{p}}{N+1}\right) \\
& \times \int_{0}^{L} d_{n_{p}}(z) e^{i\left(\Delta \beta^{(0)}-\beta_{k_{s} k_{i}}\right) z} d z .
\end{aligned}
$$

Here, $\beta_{k_{s} k_{i}}=2 C\left\{\cos \left[\pi k_{i} /(N+1)\right]+\cos \left[\pi k_{s} /(N+1)\right]\right\}$ is an extra phase-mismatch term resulting from the transverse momentum of the various supermodes, and $L$ is the total length of the array.

In practical implementations of this type of waveguide array the phase mismatch due to the individual waveguide modes is much larger than the contribution of the WGA supermodes to the phase mismatch, i.e., $\beta_{k_{s} k_{i}} \ll \Delta \beta^{(0)}$. Therefore, in Eq. (4) we can separate the integral over the total phase mismatch into slowly and quickly varying terms $\beta_{k_{s} k_{i}}$ and $\Delta \beta^{(0)}$, respectively. Under this approximation, the integral over $z$ in Eq. (4) becomes $\sum_{j} e^{-i \beta_{k_{s} k_{i}} z_{j}} \int_{j \Lambda}^{(j+1) \Lambda} d \tau d_{n_{p}}(\tau) e^{i \Delta \beta^{(0)} \tau}$, where $\Lambda$ is a length scale over which the slowly varying term does not change significantly but the quickly varying term has one or more complete periods.

We use a QPM poling structure such that $d_{n_{p}}(z)$ is a square wave with periodicity $\Lambda=2 \pi / \Delta \beta^{(0)}$. This results in the quickly varying term appearing to change linearly over length scales much longer than one period of the poling structure [19]. The rate of increase of the quickly varying term can be described by the concept of aggregate nonlinearity, the average quantity of biphoton wave function generated from a QPM poling structure with arbitrary duty cycle. The aggregate nonlinearity is defined as $D_{n_{p}}(z)=\Lambda^{-1} \int_{0}^{\Lambda} d \tau d_{n_{p}}(\tau) e^{i \Delta \beta^{(0)} \tau}$, where $\Lambda$ is the quasi-phase-matching period. Over each duty cycle the sign of $d_{n_{p}}(z)$ will change from positive to negative at the point $l_{n_{p}}(z)$. So, the aggregate nonlinearity produced by a given duty cycle is

$$
\begin{aligned}
D_{n_{p}}(z)= & \frac{d_{0}}{\Lambda} e^{i \phi_{n_{p}}(z) \Delta \beta^{(0)}} \\
& \times\left[\int_{0}^{l_{n_{p}}(z)} e^{i \Delta \beta^{(0)} \tau} d \tau-\int_{l_{n_{p}}(z)}^{\Lambda} e^{i \Delta \beta^{(0)} \tau} d \tau\right] .
\end{aligned}
$$

Here, $d_{0}$ is the absolute value of the nonlinear coefficient $d_{n_{p}}(z)$, which is unaffected by domain poling. The arbitrary phase $e^{i \phi_{n_{p}}(z) \Delta \beta^{(0)}}$ simply results from translation of each section of the poling structure with respect to the driving laser. Dividing by the poling period $\Lambda$ ensures that the aggregate nonlinearity represents the amount of biphoton wave function produced per unit length, rather than the amount produced over a whole period. Integration of (5) gives

$$
\begin{aligned}
D_{n_{p}}(z)= & \frac{2 d_{0}}{\pi} \exp \left\{i \Delta \beta^{(0)}\left[\frac{l_{n_{p}}(z)}{2}+\phi_{n_{p}}(z)\right]\right\} \\
& \times \sin \left[\Delta \beta^{(0)} \frac{l_{n_{p}}(z)}{2}\right] .
\end{aligned}
$$

Now, by varying the translation $\phi_{n_{p}}(z)$ and the length of the positive part of the duty cycle $l_{n_{p}}(z)$, we can produce an aggregate nonlinearity with any phase and with any magnitude (less than the optimal quasi-phase-matching magnitude). Hence, by combining a few sections of different duty cycles 
(a)

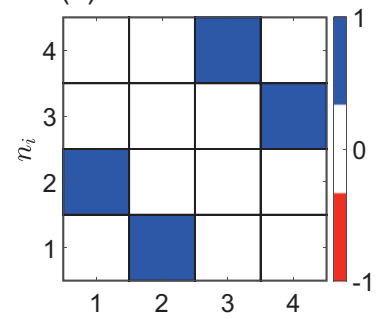

(c)

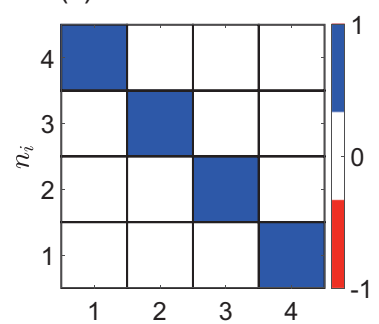

(e) $n_{s}$

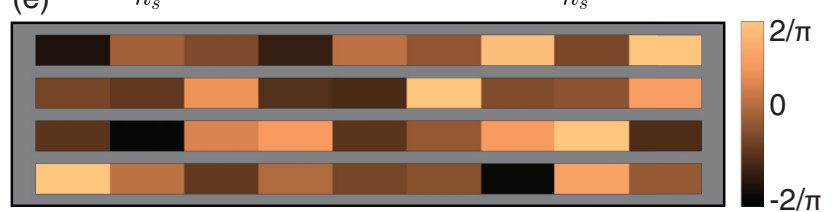

Z

FIG. 2. (Color online) (a)-(d) Target output biphoton states produced when each of the four waveguides is pumped individually, representing Bell states with dual-rail encoding. (e) The values of the aggregate nonlinear coefficient $D_{n_{p}}(z)$ along the length of each waveguide.

along the length of each waveguide we can set the aggregate nonlinearity to different values in each section [Fig. 1(b)]. This allows very flexible control over the creation of biphotons and therefore over the final output states of our device.

Substituting the aggregate nonlinearity into Eq. (4) gives

$$
\begin{aligned}
f_{k_{s}, k_{i}}(L) \approx & e^{i \beta_{k_{s} k_{i}} L} \sum_{n_{p}=1}^{N} A_{n_{p}} \sin \left(\frac{\pi k_{i} n_{p}}{N+1}\right) \sin \left(\frac{\pi k_{s} n_{p}}{N+1}\right) \\
& \times \sum_{j=1}^{M} D_{n_{p}}\left(z_{j}\right) \int_{z_{j}}^{z_{j+1}} e^{-i \beta_{k_{s} k_{i}} z} d z,
\end{aligned}
$$

which is valid in the realistic situation where $\beta_{k_{s} k_{i}} \ll \Delta \beta^{(0)}$. This equation can be inverted to solve for the aggregate nonlinearity $D_{n_{p}}\left(z_{j}\right)$, given that such a solution exists (see Sec. IV for a discussion of the existence of solutions to the inverse problem). To invert the equation, the aggregate nonlinearity is treated as a free parameter, allowed to take on a number of different values down the length of each waveguide. The number of different values of the aggregate nonlinearity is chosen to be large enough that a solution exists for the target output state $\Psi_{n_{s}, n_{i}}(L)$ when inverting Eq. (7). Generally, this will require around $N(N+1) / 2$ different values for $D_{n_{p}}\left(z_{j}\right)$ down the length of each waveguide since this is the number of free parameters in the output space.

To illustrate this general approach, we design a fourwaveguide device with poling to generate the set of twophoton Bell states as the outputs $\left|\psi_{n_{p}}\right\rangle$ [Figs. 2(a)-2(d)]. The Bell states in Figs. 2(a)-2(d) encoded with dual-rail encoding [2,12], where the signal photon occupying waveguide $1(3)$ represents a logical $O(1)$ and similarly for the idler photon in waveguides 2 and 4. Driving the waveguides simultaneously will produce a superposition of the four Bell states, with the amplitude and phase of each Bell state determined by the amplitude and phase of the classical laser driving the waveguide $n_{p}$.

We consider the device realized in lithium niobate $\left(\mathrm{LiNbO}_{3}\right)$ [15], with a waveguide length of $L=5 \mathrm{~cm}$, a coupling rate between the waveguides $C=161 \mathrm{~m}^{-1}$, and a poling period of $\Lambda=18.5 \mu \mathrm{m}$ at $230^{\circ} \mathrm{C}$ for $775 \mathrm{~nm}$ pump wavelength. Using these parameters we solve Eq. (7) for the four Bell states [Figs. 2(a)-2(d)]; this provides the required aggregate nonlinearities in the four-waveguide array [Fig. 2(e)]. From the values of the aggregate nonlinearity we can reconstruct the full poling structure $d_{n_{p}}(z)$, using Eq. (6), the reconstruction is shown in Fig. 3(e). To check our solutions numerically, we compare the states produced by the full poling structure to the target Bell states; the errors are shown in Figs. 3(a)-3(d). The fidelities between the target states and the realized states are all greater than 0.999; this shows that (a)

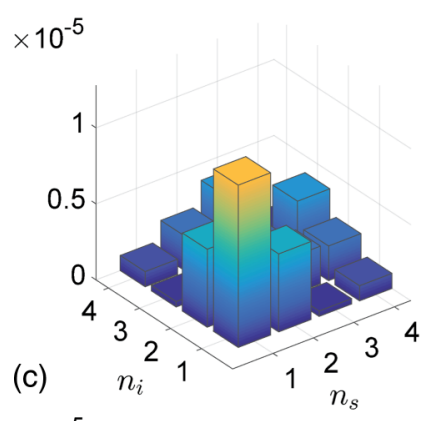

$\times 10^{-5}$

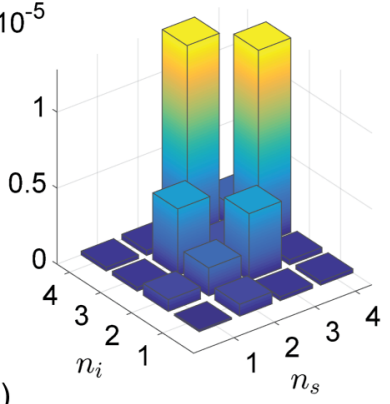

(b) $\times 10^{-5}$

(d)

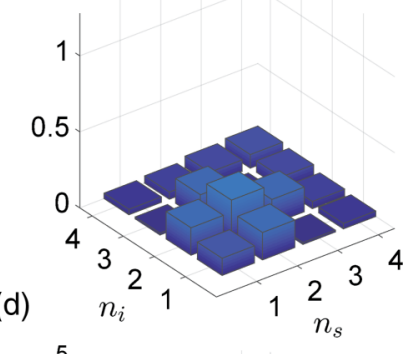

$\times 10^{-5}$

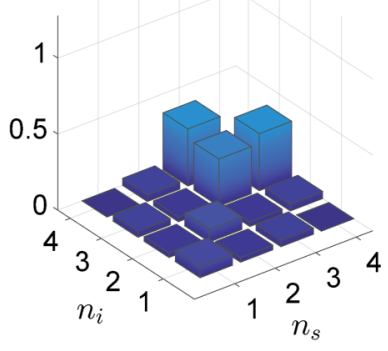

(e)

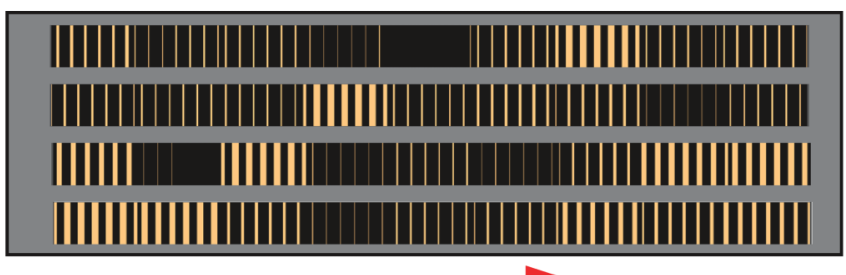

Z

FIG. 3. (Color online) (a)-(d) The error between the target states in Figs. 2(a)-2(d), and the states resulting from using the poling structures in (e). (e) The poling structures used to approximate the aggregate nonlinearities in Fig. 2(e); two colors represent the up and down orientations of the ferroelectric dipole moment. The number of domains has been reduced to approximately 100 for visualization. 
the approximate use of the aggregate nonlinear coefficient is valid for realistic parameters.

To conclude this section, we have demonstrated how to create all-optically-reconfigurable linear combinations of the set of two-photon Bell states in an array of four coupled nonlinear waveguides with special poling. Moreover, the poling technique can be applied to a WGA to enable it to produce any set of biphoton states. This opens the door for the design of a variety of reconfigurable entangled photon sources, with output quantum spaces tailored to specific technological applications. We also note that the nearest-neighbor coupling interactions and nonlinear effects we consider here are common to many physical systems. For instance, similar alloptically-reconfigurable control of entangled photons could be achieved via spontaneous four-wave mixing in $\chi^{(3)}$ media.

\section{EFFECT OF DEGENERACIES ON AVAILABLE OUTPUT SPACE}

We have shown that a nonlinear four-waveguide waveguide array can be used to generate any linear combination of the set of Bell states. In fact, domain poling patterns in waveguide arrays provide nearly limitless freedom to produce different quantum states. This, combined with WGA's dynamic all-optical reconfigurability, provides a very flexible source of quantum states of light.

To design a waveguide array capable of being optically reconfigured over a given output space, the array must be able to produce the set of "basis" output states $\left|\psi_{n_{p}}\right\rangle$, that define the full output space. If these states can be produced from the device by only varying the pumping lasers, then the device can be reconfigured over the full subspace to any state $|\Psi\rangle=\sum_{n_{p}} A_{n_{p}}\left|\psi_{n_{p}}\right\rangle$. As we will show in this section, degeneracies in WGA's transverse modes mean that not every subspace defined by a set of arbitrary $\left|\psi_{n_{p}}\right\rangle$ can be spanned. In Sec. V, we show that this limitation is removed if the degeneracies are broken by introducing refractive index differences between waveguides.

To investigate the effect of degeneracies on the output space of a WGA, it is convenient to consider using domain poling structures in the WGA with different poling periods in different locations. Localized adjustment of the poling period allows phase matching with particular transverse modes of the array. This lets us to isolate and drive each transverse mode in different sections of the array, thus allowing any linear combination of these modes to be produced at the output of the array. Hence, any quantum state can be produced at the end of the array given that all the transverse modes can be driven individually. To demonstrate this we again consider Eq. (2) from Sec. II, showing the output from the array in terms of transverse modes $f_{k_{s}, k_{i}}$ :

$$
\begin{aligned}
f_{k_{s}, k_{i}}(L)= & e^{i \beta_{k_{s} k_{i}} L} \sum_{n_{p}=1}^{N} A_{n_{p}} \sin \left(\frac{\pi k_{i} n_{p}}{N+1}\right) \sin \left(\frac{\pi k_{s} n_{p}}{N+1}\right) \\
& \times \int_{0}^{L} d_{n_{p}}(z) e^{i\left(\Delta \beta^{(0)}-\beta_{k_{s} k_{i}}\right) z} d z
\end{aligned}
$$

Here, we see that poling the nonlinear coefficient, $d_{n_{p}}(z)$ with a frequency of $\Delta \beta^{(0)}-\beta_{k_{s}^{\prime} k_{i}^{\prime}}$ will selectively drive trans-

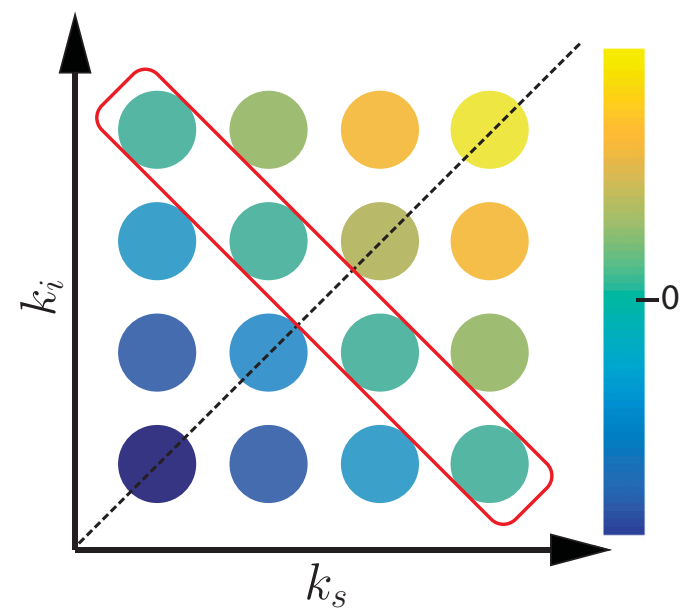

FIG. 4. (Color online) Two-photon eigenvalues $\left(\beta_{k_{i} k_{s}}\right)$ of the transverse modes for a four-waveguide array. The dashed line shows the symmetry axis $k_{i}=k_{s}$. The diagonal rectangle marks the degenerate eigenvalues along the line $k_{s}=N+1-k_{i}$.

verse modes that have propagation constants $\beta_{k_{s}^{\prime}, k_{i}^{\prime}}$. Hence, transverse modes with unique propagation constants can be phase matched and driven individually, without affecting other modes. In order to determine which modes have unique propagation constants, we must look at the spectrum of the modes' eigenvalues as a function of $k_{i}$ and $k_{s}$. This spectrum is given by

$$
\beta_{k_{i} k_{s}}=2 C\left[\cos \left(\frac{\pi k_{i}}{N+1}\right)+\cos \left(\frac{\pi k_{s}}{N+1}\right)\right],
$$

and a plot of the modes' propagation constants for $N=4$ is shown in Fig. 4. First it is important to note that the equation is transcendental, so there will be some randomly occurring degeneracies, especially for large $N$. There will also be some degeneracies that can be predicted analytically. Here, we will consider only the degeneracies that can be predicted analytically since in WGA's with only a few waveguides these are by far the most common type of degeneracy.

The most obvious of these predictable degeneracies occurs because the signal and idler are almost degenerate, so naturally $\beta_{k_{i} k_{s}}=\beta_{k_{s} k_{i}}$, and the eigenvalues are symmetric across the main diagonal. This degeneracy is not an obstacle to showing that the device can generate any quantum state because it arises from the particles themselves rather than from the WGA. However, there will also be degenerate eigenvalues when $k_{s}=N+1-k_{i}$ since in this case $\beta_{k_{i},\left(N+1-k_{i}\right)}=0$ for all $k_{i}$. This degeneracy corresponds to the set of modes where the entangled signal and idler photons have equal and opposite propagation constants, so the net propagation constant of the state is zero. This presents a problem because now the degenerate eigenvalues correspond to distinguishable states, but we cannot drive these states independently with domain poling.

As a result of the degeneracy of the $f_{k_{i},\left(N+1-k_{i}\right)}$ modes, just choosing the domain poling period cannot drive the modes individually. This limitation can be overcome by adjusting the pump intensity in each waveguide. The degenerate transverse modes $f_{k_{i},\left(N+1-k_{i}\right)}$ have distinct spatial profiles for each $k_{i}$. 
Hence, shaping the spatial profile of the laser driving of the device can drive one of the transverse modes without exciting the others. Coupling the pump laser into different waveguides will change the rate each of the modes $f_{k_{i} k_{s}}$ is driven at. We will call the rate each mode is driven at $P_{k_{i} k_{s}}$, the pump profile. From Eq. (8) we can see the pump profile is given by

$$
P_{k_{i} k_{s}}=\sum_{n_{p}=1}^{N} A_{n_{p}} \sin \left(\frac{\pi k_{i} n_{p}}{N+1}\right) \sin \left(\frac{\pi k_{s} n_{p}}{N+1}\right),
$$

where $n_{p}$ denotes the waveguide numbers the pumping lasers are coupled to. Now, to control the driving rate of modes with degenerate eigenvalues we should look at $P_{k_{i}\left(N+1-k_{i}\right)}$ :

$$
\begin{aligned}
& P_{k_{i}\left(N+1-k_{i}\right)} \\
& \quad=\sum_{n_{p}=1}^{N} A_{n_{p}} \sin \left(\frac{\pi k_{i} n_{p}}{N+1}\right) \sin \left[\frac{\pi\left(N+1-k_{i}\right) n_{p}}{N+1}\right] .
\end{aligned}
$$

This is the rate that each of the degenerate modes $f_{k_{i},\left(N+1-k_{i}\right)}$ is driven at when pumping each waveguide with an arbitrary intensity and phase pump laser $A_{n_{p}}$. It is equivalent to

$$
P_{k_{i}\left(N+1-k_{i}\right)}=-\frac{1}{2} \sum_{n_{p}=1}^{N} A_{n_{p}}(-1)^{n_{p}}\left[1-\cos \left(\frac{2 \pi k_{i} n_{p}}{N+1}\right)\right] .
$$

Now, if we drive each waveguide with laser field given by $A_{n_{p}}=\cos \left[2 \pi k_{p} n_{p} /(N+1)\right](-1)^{n_{p}}$, then $P_{k_{i}\left(N+1-k_{i}\right)}=$ $\delta_{k_{i} k_{p}}+\delta_{\left(N+1-k_{i}\right) k_{p}}$. Here, $k_{p}$ denotes the mode number of the pump. This pump profile allows the mode $f_{k_{i}^{\prime}}\left(N+1-k_{i}^{\prime}\right)$ to be addressed individually, without exciting the other modes sharing its degenerate propagation constant. By using a linear combination of these pump profiles, i.e.,

$$
A_{n_{p}}=\sum_{k_{i}} c_{k_{i}} \cos \left(\frac{2 \pi k_{p} n_{p}}{N+1}\right)(-1)^{n_{p}}
$$

any linear combination of the degenerate modes can be driven. Therefore, the degeneracy in the modes' propagation constants can be overcome by carefully shaping the pumping $A_{n_{p}}$ to exploit the spatial differences between degenerate modes.

Importantly pumping with the profiles $A_{n_{p}}=$ $\sin \left[2 \pi k_{p} n_{p} /(N+1)\right](-1)^{n_{p}}$ never excites the degenerate $f_{k_{i}\left(N+1-k_{i}\right)}$ modes. So, this gives $N / 2$ degrees of freedom for exciting other modes while simultaneously using the $\cos \left[2 \pi k_{p} n_{p} /(N+1)\right](-1)^{n_{p}}$ pumping profile to excite the $N / 2$ degenerate $f_{k_{i}\left(N+1-k_{i}\right)}$ modes. This could be useful for allowing reconfigurability, despite degeneracies, by appropriate shaping of the poling structures in the array.

To summarize, we have shown in this section that waveguide arrays can be designed to span large quantum output spaces via all-optical reconfigurability, even in the presence of degeneracies. Through careful choice of which waveguide is driven to produce which basis state $\left|\psi_{n_{p}}\right\rangle$, the desired output space can often be spanned. For example, in the preceding section we show that the set of four Bell states can be spanned with pump excitation of individual waveguides. (a)

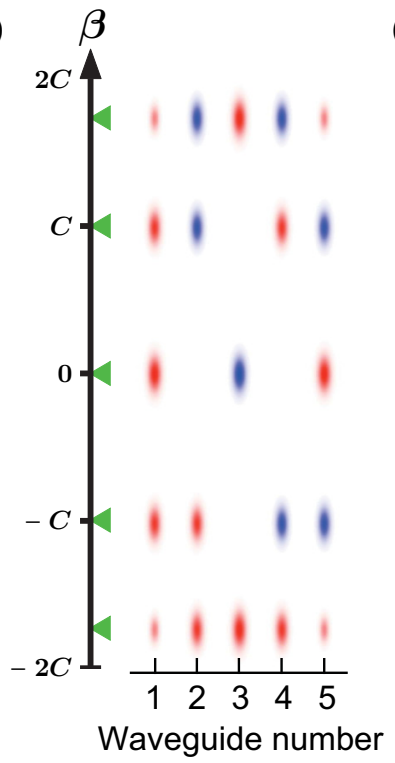

(b)

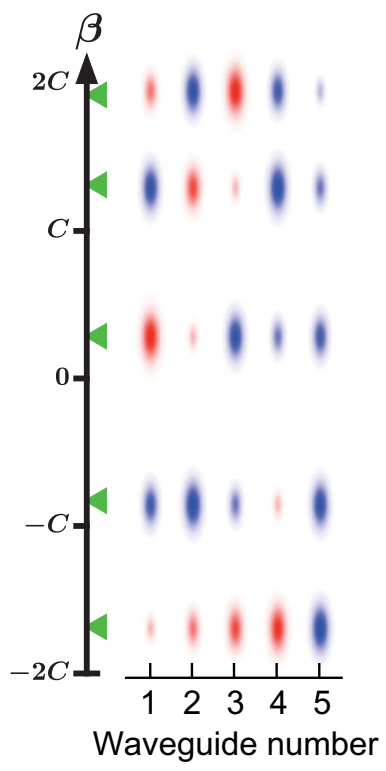

FIG. 5. (Color online) Single-photon mode profiles and their corresponding propagation constants for arrays of five waveguides with (a) the same refractive index in all waveguides, (b) with one of the edge waveguides having a higher refractive index.

\section{REMOVING DEGENERACIES FOR MAXIMUM RECONFIGURABILITY}

We now consider how degeneracies could be removed from a nonlinear waveguide array to allow it to span any $N$-dimensional biphoton output space. Although (as shown in Sec. II) arrays with small numbers of waveguides can span interesting output spaces such as the Bell states, in principle not every output space can be spanned due to degeneracies. This becomes more important for large numbers of waveguides because the increasing numbers of modes increase the probability that the transcendental equation for the eigenmodes [Eq. (9)] will produce more degeneracies.

Degeneracies occur when the difference between two single-photon modes' eigenvalues are the same. This can be shown by assuming a pair of two-photon modes are degenerate,

$$
\beta_{k_{s_{1}}, k_{i_{1}}}=\beta_{k_{s_{2}}, k_{i_{2}}} \Rightarrow \beta_{k_{s_{1}}}+\beta_{k_{i_{1}}}=\beta_{k_{s_{2}}}+\beta_{k_{i_{2}}},
$$

then inverting to find a condition relating to the difference between eigenvalues of single-photon modes

$$
\beta_{k_{s_{1}}}-\beta_{k_{s_{2}}}=\beta_{k_{i_{2}}}-\beta_{k_{i_{1}}} \text {. }
$$

So, to remove degeneracies we require that all the differences between eigenvalues of single-photon modes $\beta_{k_{s_{1}}}-\beta_{s_{s_{2}}}$ are not equal. This could be achieved by tuning the refractive index in each waveguide to ensure Eq. (15) is violated for all single-photon modes.

In Fig. 5, we give an example of this degeneracy breaking for an array of five waveguides. The removal of degeneracies can be seen by noting the nonuniform spacing of singlephoton modes propagation constants in Fig. 5(b), meaning the condition for degeneracy [Eq. (15)] will not be fulfilled. This shows that a small adjustment in refractive index can remove degenerate propagation constants as well as removing zero points from transverse modes. 
Allowing for different refractive indices in all waveguides the propagation of biphotons is governed by the following equation:

$$
\begin{aligned}
i \frac{\partial \Psi_{n_{s}, n_{i}}(z)}{\partial z}= & i \sum_{n_{p}=1}^{N} A_{n_{p}} d_{n_{p}}(z) e^{i \Delta \beta^{(0)} z} \delta_{n_{s}, n_{p}} \delta_{n_{i}, n_{p}} \\
& -C\left[\Psi_{n_{s}, n_{i}+1}+\Psi_{n_{s}, n_{i}-1}+\Psi_{n_{s}+1, n_{i}}+\Psi_{n_{s}-1, n_{i}}\right] \\
& +\left(\delta \beta_{n_{s}}+\delta \beta_{n_{i}}\right) \Psi_{n_{s}, n_{i}},
\end{aligned}
$$

where $\delta \beta_{n_{s}}+\delta \beta_{n_{i}}$ is the sum of the propagation constants due to refractive index modulation in the waveguides occupied the by signal and idler photons. We now introduce a new set of transverse single-photon modes $u_{n}^{(k)}$ to account for the new propagation constants in the array. These are eigenmodes of the combined single-photon coupling and propagation operators from Eq. (16), so for the $k$ th mode

$$
\beta_{k} u_{n}^{(k)}=-C\left[u_{n+1}^{(k)}+u_{n-1}^{(k)}\right]+\delta \beta_{n} u_{n}^{(k)} .
$$

The two-photon wave function produced by down conversion can be expressed in terms of pairs of single-photon modes such that $\Psi_{n_{s}, n_{i}}=\sum_{k_{s}, k_{i}} f_{k_{s}, k_{i}} u_{n_{s}}^{\left(k_{s}\right)} \otimes u_{n_{i}}^{\left(k_{i}\right)}$. Writing Eq. (16) in terms of $f_{k_{s}}, k_{i}$ gives

$$
i \frac{\partial f_{k_{s}, k_{i}}(z)}{\partial z}=i D_{k_{s}, k_{i}}+\beta_{k_{s}, k_{i}} f_{k_{s}, k_{i}}(z) .
$$

Here, $\beta_{k_{s}, k_{i}}=\left(\beta_{k_{s}}+\beta_{k_{i}}\right)$ and $D_{k_{s}, k_{i}}$ is the spatial overlap between the pumping and the real-space profile of the two-photon mode $D_{k_{s}, k_{i}}=\sum_{n_{p} n_{s} n_{i}}^{N} A_{n_{p}} u_{n_{s}}^{\left(k_{s}\right)} \otimes$ $u_{n_{i}}^{\left(k_{i}\right)} \delta_{n_{i}, n_{p}} \delta_{n_{s}, n_{p}} d_{n_{p}}(z) e^{i \Delta \beta^{(0)} z}$. The solution to Eq. (18) is

$$
\begin{aligned}
f_{k_{s}, k_{i}}(L)= & e^{i \beta_{k_{s}, k_{i}} L} \sum_{n_{p}}^{N} A_{n_{p}} u_{n_{p}}^{\left(k_{s}\right)} \\
& \otimes u_{n_{p}}^{\left(k_{i}\right)} \int_{0}^{L} d_{n_{p}}(z) e^{i\left(\Delta \beta^{(0)}-\beta_{k_{s}, k_{i}}\right) z} .
\end{aligned}
$$

This is analogous to Eq. (8), but now with a new set of modes and eigenvalues. From this equation, the two conditions to exclusively drive any two-photon mode in any waveguide are evident:

(1) The mode must have a unique eigenvalue $\beta_{k_{s}, k_{i}}$, so that mode can be selectively driven by poling at the resonant spatial frequency $\Delta \beta^{(0)}-\beta_{k_{s}, k_{i}}$.

(2) It must be possible to drive the mode by pumping any waveguide, so the overlap of the mode $D_{k_{s}, k_{i}}$ must be nonzero, when each waveguide is driven individually.

The first condition ensures that each mode can be driven exclusively, while the second condition ensures that this exclusive driving can occur in any waveguide. In Fig. 5 it is shown that both criteria are satisfied for an array of five waveguides, but only after one waveguide is given a different refractive index to the others, so that $\delta \beta_{n}=1$ for one waveguide in Eq. (16). These two criteria make it possible to design a WGA to span any desired $N$-dimensional output space.

This would be achieved by poling each waveguide with $N(N+1) / 2$ different segments of poling. Each segment being poled with a period $2 \pi /\left(\Delta \beta^{(0)}+\beta_{k_{s}, k_{i}}\right)$ to phase match a particular mode. If the length of each segment is set so that $L \gg 1 / \min \left(\left|\beta_{k_{s}, k_{i}}-\beta_{k_{s}, k_{i}}\right|\right)$, then only the mode that is phase matched will be produced in a particular segment. Then, by varying the relative length of each segment, any linear combination of modes can be produced at the end of the array. This process could be used in every waveguide, engineering each to produce a different state $\psi_{n_{p}}$. Then, driving the waveguides simultaneously can span the entire space defined by the $\psi_{n_{p}}$. Since the $\psi_{n_{p}}$ can now, in the absence of degeneracies, be set to any state this means that the array can now be engineered to be all-optically reconfigured over any $N$-dimensional subspace.

So, in conclusion, waveguide arrays can be engineered to span any $N$-dimensional quantum output space. However, this will generally require some optimization of the refractive index in each waveguide in order to remove degeneracies.

\section{INFERRING THE BIPHOTON WAVE FUNCTION FROM CLASSICAL MEASUREMENTS}

Fabrication of complex domain poling patterns in waveguide array devices will no doubt involve some systematic and random errors. Typically large numbers of devices with varied parameters will be fabricated on a single chip, and then the quantum properties of each device will be characterized. The task of characterizing each device is very time consuming due to the difficulty of the quantum correlation measurements and the shear number of devices to be characterized. In order to efficiently test which devices operate correctly and which have unacceptably large fabrication errors, one can use the "stimulated emission tomography" [24] approach based on classical difference frequency generation (DFG) as an alternative to measurements of the quantum correlations caused by SPDC. This would provide a quick and efficient way of separating defective devices from correctly operating ones. Then, further more detailed characterization can be performed on devices that pass the initial classical DFG testing.

Here, we show how to use classical difference frequency generation in a waveguide array to determine the quantum wave function that would be produced by SPDC. Spontaneous parametric down conversion is the quantum analog of difference frequency generation. The key difference being that DFG is stimulated by a specific photon state (the seed laser) where as SPDC is stimulated via quantum vacuum fluctuations. Remarkably, it is possible to reconstruct the biphoton wave function that would be produced by SPDC by carefully choosing the seed field of DFG, as is demonstrated in Ref. [24]. We show how to apply this technique to arrays of coupled waveguides, allowing characterization of the SPDC wave function using classical DFG measurements.

First, we derive the equation for DFG in an array of coupled waveguides. Then, we show how seeding the array with a specific seed field profile allows reconstruction of an idler field proportional to the SPDC wave function. Finally, we demonstrate that a complex seed profile is not actually needed to simulate SPDC. We can instead simply seed one waveguide at a time, then add a linear combination of the output idler fields together to achieve the same output that would be produced by the seed with complex spatial profile. This is due to the 
linearity of idler field with respect to the seed field in the case of negligible pump depletion.

\section{A. Difference frequency generation in a waveguide array}

The equation for the idler field produced by DFG in an array of coupled waveguides is

$$
\begin{aligned}
i \frac{\partial E_{n_{i}}}{\partial z}= & -C\left[E_{n_{i}+1}+E_{n_{i}-1}\right] \\
& +i E_{n_{s}}^{(s)^{*}} A_{n_{p}} d_{n_{p}}(z) e^{i \Delta \beta^{(0)} z} \delta_{n_{i}, n_{p}} \delta_{n_{s}, n_{p}},
\end{aligned}
$$

where we assume the fields of the pump $\left(A_{n_{p}}\right)$, and seed $\left(E_{n_{s}}^{(s)}\right)$ lasers are undepleted, and also that only one waveguide $\left(n_{p}\right)$ is driven by the pump laser. We can solve this equation in the same way as for the SPDC case. Using reciprocal space defined by

$$
E_{n_{i}}=\sum_{k_{i}=1}^{N} \sin \left(\frac{\pi k_{i} n_{i}}{N+1}\right) f_{k_{i}}
$$

we get

$$
\begin{aligned}
f_{k_{i}}(L)= & e^{\left(i \beta_{k_{i}} L\right)} \int_{0}^{L} d z e^{\left[i\left(\Delta \beta^{(0)}-\beta_{k_{i}}\right) z\right]} \\
& \times \sin \left(\frac{\pi k_{i} n_{p}}{N+1}\right) E_{n_{p}}^{(s)^{*}} A_{n_{p}} d_{n_{p}}(z) .
\end{aligned}
$$

Here, we have introduced $\beta_{k_{i}}=2 C \cos \left[\pi k_{i} /(N+1)\right]$, the contribution of the idler photons transverse modes to the phase-matching conditions.

The seed field will also couple into neighboring waveguides in the array, so the transverse profile of $E_{n_{s}}^{(s)}$ will evolve according to

$$
i \frac{\partial E_{n_{s}}^{(s)}}{\partial z}=-C\left[E_{n_{s}+1}^{(s)}+E_{n_{s}-1}^{(s)}\right] .
$$

This is easily solved using the same method as was used to solve Eq. (20) giving

$$
f_{k_{s}}(z)=f_{k_{s}}(0) \exp \left[2 i C \cos \left(\frac{\pi k_{s}}{N+1}\right) z\right]
$$

in reciprocal space, and in position space

$$
\begin{aligned}
E_{n_{s}}^{(s)}(z)= & \frac{2}{N+1} \sum_{k_{s}=1}^{N} \sin \left(\frac{\pi k_{s} n_{s}}{N+1}\right) f_{k_{s}}(0) \\
& \times \exp \left[2 i C \cos \left(\frac{\pi k_{s}}{N+1}\right) z\right] .
\end{aligned}
$$

Now, we can substitute this profile for the seed field $E_{n_{s}}^{(s)}(z)$ into the expression for the idler field (22). This gives

$$
\begin{aligned}
f_{k_{i}}^{(\mathrm{DFG})}(L)= & \frac{2}{N+1} e^{i \beta_{k_{i}} L} \sum_{k_{s}=1}^{N} \int_{0}^{L} d z e^{i\left(\Delta \beta^{(0)}-\beta_{k_{i}}-\beta_{k_{s}}\right) z} \\
& \times \sin \left(\frac{\pi k_{i} n_{p}}{N+1}\right) \sin \left(\frac{\pi k_{s} n_{p}}{N+1}\right) f_{k_{s}}^{(s)}(0)^{*} A_{n_{p}} d_{n_{p}}(z),
\end{aligned}
$$

with $\beta_{k_{i}}=2 C \cos \left[\pi k_{i} /(N+1)\right]$ and $\beta_{k_{s}}=2 C \cos \left[\pi k_{s} /\right.$ $(N+1)]$. Shown in Fig. 6 is an example of the propagation of

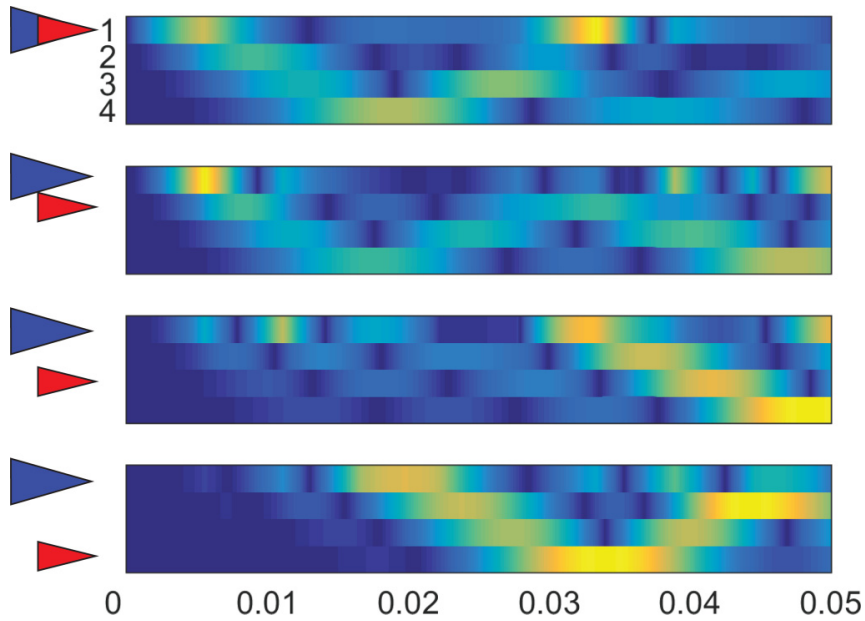

FIG. 6. (Color online) Idler field intensity $\left(\left|E_{n_{i} n_{s^{\prime}}}\right|^{2}\right)$ evolution along the waveguides in the process of difference frequency generation. The larger arrow shows the pumped waveguide $n_{p}$, while the smaller arrow shows the seeded waveguide $n_{s}^{\prime}$.

the idler field during DFG in the nonlinear WGA [poled with the patterns from Fig. 3(e)]. Here, the seed field is coupled into each waveguide in turn while the pump waveguide remains coupled to the first waveguide. Then, the output idler fields provide information about the quantum state that would be produced via SPDC when pumping the first waveguide.

At this point, it is interesting to compare the classical signal field $f_{k_{i}}$ to the wave function for the SPDC case [Eq. (4)]

$$
\begin{aligned}
f_{k_{s}, k_{i}}^{(\mathrm{SPDC})}(L)= & e^{i\left(\beta_{k_{i}}+\beta_{k_{s}}\right) L} \int_{0}^{L} d z e^{i\left(\Delta \beta^{(0)}-\beta_{k_{i}}-\beta_{k_{s}}\right) z} \\
& \times \sin \left(\frac{\pi k_{i} n_{p}}{N+1}\right) \sin \left(\frac{\pi k_{s} n_{p}}{N+1}\right) A_{n_{p}} d_{n_{p}}(z)
\end{aligned}
$$

Equations (27) and (26) are very similar, except in the classical DFG case all the seed modes $k_{s}$ are summed over, eliminating the correlation between signal and idler. Now, in Eq. (26) we can set $f_{k_{s}}^{(s)}(0)=\delta_{k_{s}, k_{s^{\prime}}}$ so that the input seed is in some eigenstate of the coupling operator. This means that the output DFG state will be proportional to the SPDC state $f_{k_{s^{\prime}}, k_{i}}^{\text {(SPDC) }}(L)$. So, by making $N$ measurements of the DFG output for $k_{s^{\prime}}=1,2, \ldots, N$ we can construct the full SPDC wave function $f_{k_{s}, k_{i}}^{(\text {SPDC) }}(L)$. Also, we give the initial seed mode a specific phase so that it matches the SPDC equation, hence, the required seed profile is $f_{k_{s}}^{(s)}(0)=f_{0}^{(s)} \delta_{k_{s}, k_{s^{\prime}}} e^{\left(-i \beta_{k_{s}} L\right)}$, and this gives

$$
\begin{aligned}
f_{k_{i}}^{(\mathrm{DFG})}(L)= & \frac{2 e^{\left[i\left(\beta_{k_{i}}+\beta_{k_{s}}\right) L\right]}}{N+1} \int_{0}^{L} d z e^{\left[i\left(\Delta \beta^{(0)}-\beta_{k_{i}}-\beta_{k_{s}}\right) z\right]} \\
& \times \sin \left(\frac{\pi k_{i} n_{p}}{N+1}\right) \sin \left(\frac{\pi k_{s} n_{p}}{N+1}\right) A_{n_{p}} f_{0}^{(s)} d_{n_{p}}(z),
\end{aligned}
$$

which is identical to Eq. (27) up to a constant factor $2 f_{0}^{(s)} /$ $(N+1)$. 

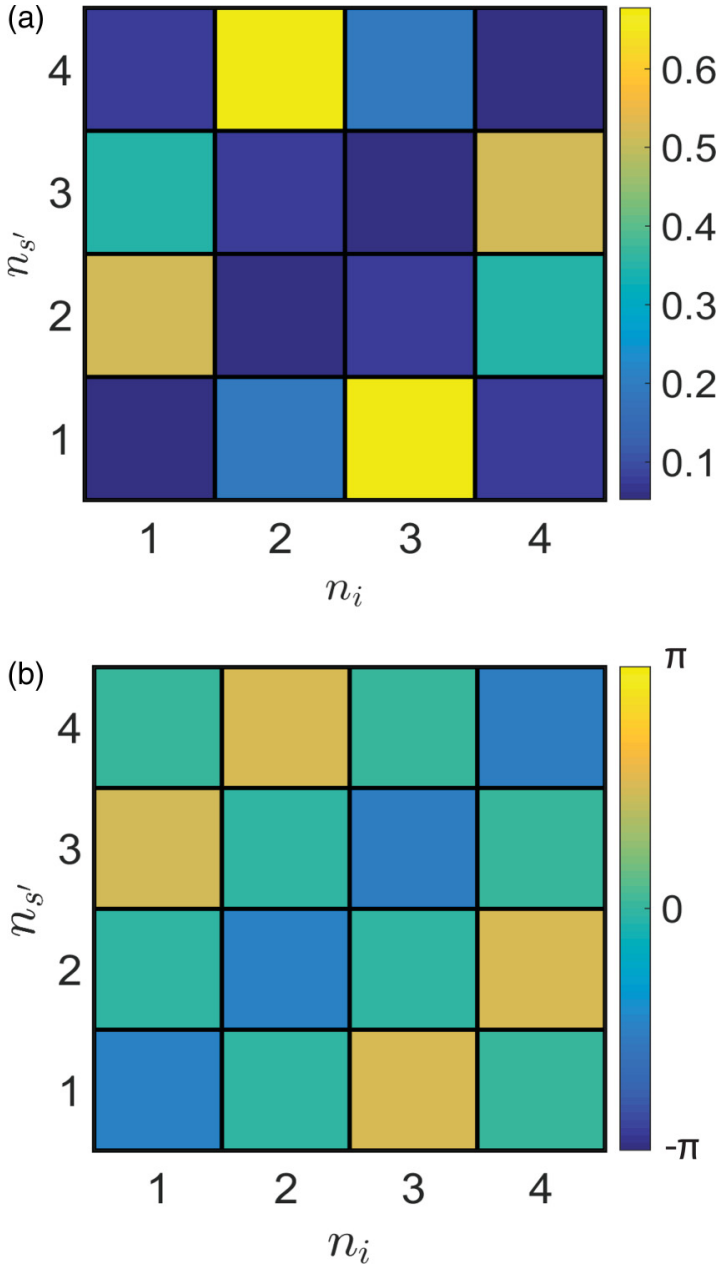

FIG. 7. (Color online) Output idler field: (a) intensity $\left(\left|E_{n_{i} n_{s^{\prime}}}\right|^{2}\right)$ and (b) phase of $E_{n_{i} n_{s^{\prime}}}$. Each row corresponds the seed beam being coupled into a different waveguide $n_{s^{\prime}}$.

\section{B. Reconstruction of $\Psi^{(\mathrm{SPDC})}$ from $E^{(\mathrm{DFG})}$}

We have shown that DFG with the seed pulse in a particular transverse mode will be proportional to one row of the SPDC wave function (a phase factor is also required). So, by taking multiple measurements of DFG output, with the seed in a different mode each time, it is possible to reconstruct the complete quantum mechanical wave function produced by SPDC. However, in practice it would be difficult to couple a seed laser into a waveguide array in a specific transverse mode.

Typically, the easiest quantity to measure would be the output idler field produced when seeding only one waveguide and pumping only one waveguide. We will denote the measured idler output field $E_{n_{i} n_{\mathrm{s}^{\prime}}}$, where $n_{i}$ is the waveguide number the idler field is measured at and $n_{s}^{\prime}$ is the waveguide that the input seed field was coupled into. Taking multiple measurements of $E_{n_{i} n_{s^{\prime}}}$, but with the seed laser coupled into a different waveguide each time (while the pump remains in waveguide $n_{p}$ ) will produce an $N \times N$ matrix (Fig. 7). As we will show, this matrix provides enough information to determine the quantum state that would be produced via SPDC when pumping the waveguide $n_{p}$. We will assume that this $N \times N$ matrix is the quantity that is actually measured, and we will show how to reconstruct the SPDC wave function from the measured values.

We denote the $k$-space form of $E_{n_{i} n_{s^{\prime}}}$ as $f_{k_{i}, n_{s^{\prime}}}^{\text {(meas.) }}(L)$, where $k_{i}$ is the transverse mode of the output idler field, and $n_{s^{\prime}}$ remains as the waveguide number that the seed laser is coupled to. The expression for $f_{k_{i}, n_{s^{\prime}}}^{\text {(meas.) }}(L)$ can be found from Eq. (26) by setting the initial seed profile to $E_{n_{s}}^{(s)}(0)=\delta_{n_{s}, n_{s^{\prime}}}$ so that only waveguide $n_{s^{\prime}}$ is seeded. This gives

$$
\begin{aligned}
f_{k_{i}, n_{s^{\prime}}}^{(\text {meas })}(L)= & \frac{2}{N+1} e^{\left(i \beta_{k_{i}} L\right)} \\
& \times \sum_{k_{s}=1}^{N} \sin \left(\frac{\pi k_{i} n_{p}}{N+1}\right) \sin \left(\frac{\pi k_{s} n_{p}}{N+1}\right) \sin \left(\frac{\pi k_{s} n_{s^{\prime}}}{N+1}\right) \\
& \times \int_{0}^{L} d z e^{\left[i\left(\Delta \beta^{(0)}-\beta_{k_{i}}-\beta_{k_{s}}\right) z\right]} A_{n_{p}} d_{n_{p}}(z) .
\end{aligned}
$$

Note that setting $E_{n_{s}}^{(s)}(0)=\delta_{n_{s}, n_{s^{\prime}}}$ implies that the seed magnitude and phase remain the same when changing the seed laser to different waveguides $n_{s^{\prime}}$ to measure different elements of $f_{k_{i}, n_{s^{\prime}}}^{\text {(meas) }}(L)$. In general, it is not necessary to keep the intensity and phase the same, but any variations must be known to reconstruct the SPDC wave function. Here, we assume for simplicity that the phase and intensity of the seed remains constant regardless of which waveguide is seeded.

Now, once the idler output has been measured with the seed in each of the $N$ waveguides, the SPDC wave function can be mathematically reconstructed from the measured values. This gives a reconstructed function $f_{k_{i}, k_{s^{\prime}}}^{\text {(recon.) }}(L)$ which is proportional to the SPDC wave function in Eq. (27):

$$
f_{k_{i}, k_{s^{\prime}}}^{(\text {recon })}(L)=e^{\left(i \beta_{k_{s^{\prime}}} L\right)} \sum_{n_{s^{\prime}}=1}^{N} \sin \left(\frac{\pi n_{s^{\prime}} k_{s^{\prime}}}{N+1}\right) f_{k_{i}}^{\text {(meas) }}(L) .
$$

Here, we add together a superposition on the measured outputs, weighted by a sin function, and multiplied by a phase factor $e^{\left(i \beta_{k_{s^{\prime}}} L\right)}$. The $k_{s^{\prime}}$ argument determines which column of the SPDC wave function is reconstructed. To confirm that $f_{k_{i}, k_{s^{\prime}}}^{\text {(recon) }}(L)$ is a reconstruction of the SPDC wave function, we substitute in the full equation for $f_{k_{i}, n_{s^{\prime}}}^{(\text {meas) }}(L)$, then simplify

$$
\begin{aligned}
f_{k_{i}, k_{s^{\prime}}}^{(\text {recon })}(L)= & e^{\left(i \beta_{k_{s^{\prime}}} L\right)} \sum_{n_{s^{\prime}}=1}^{N} \sin \left(\frac{\pi n_{s^{\prime}} k_{s^{\prime}}}{N+1}\right) \frac{2}{N+1} e^{\left(i \beta_{k_{i}} L\right)} \\
& \times \sum_{k_{s}=1}^{N} \sin \left(\frac{\pi k_{i} n_{p}}{N+1}\right) \sin \left(\frac{\pi k_{s} n_{p}}{N+1}\right) \sin \left(\frac{\pi k_{s} n_{s^{\prime}}}{N+1}\right) \\
& \times \int_{0}^{L} d z e^{\left[i\left(\Delta \beta^{(0)}-\beta_{k_{i}}-\beta_{k_{s}}\right) z\right]} A_{n_{p}} d_{n_{p}}(z)
\end{aligned}
$$

summing over the seeded waveguide number $n_{s^{\prime}}$ :

$$
\begin{aligned}
f_{k_{i}, k_{s^{\prime}}}^{(\mathrm{recon})}(L)= & e^{\left(i \beta_{s^{\prime}} L\right)} \frac{2}{N+1} e^{\left(i \beta_{k_{i}} L\right)} \\
& \times \sum_{k_{s^{\prime}}=1}^{N} \sin \left(\frac{\pi k_{i} n_{p}}{N+1}\right) \sin \left(\frac{\pi k_{s^{\prime}} n_{p}}{N+1}\right) \\
& \times \int_{0}^{L} d z e^{\left[i\left(\Delta \beta^{(0)}-\beta_{k_{i}}-\beta_{k_{s^{\prime}}}\right) z\right]} A_{n_{p}} d_{n_{p}}(z) .
\end{aligned}
$$



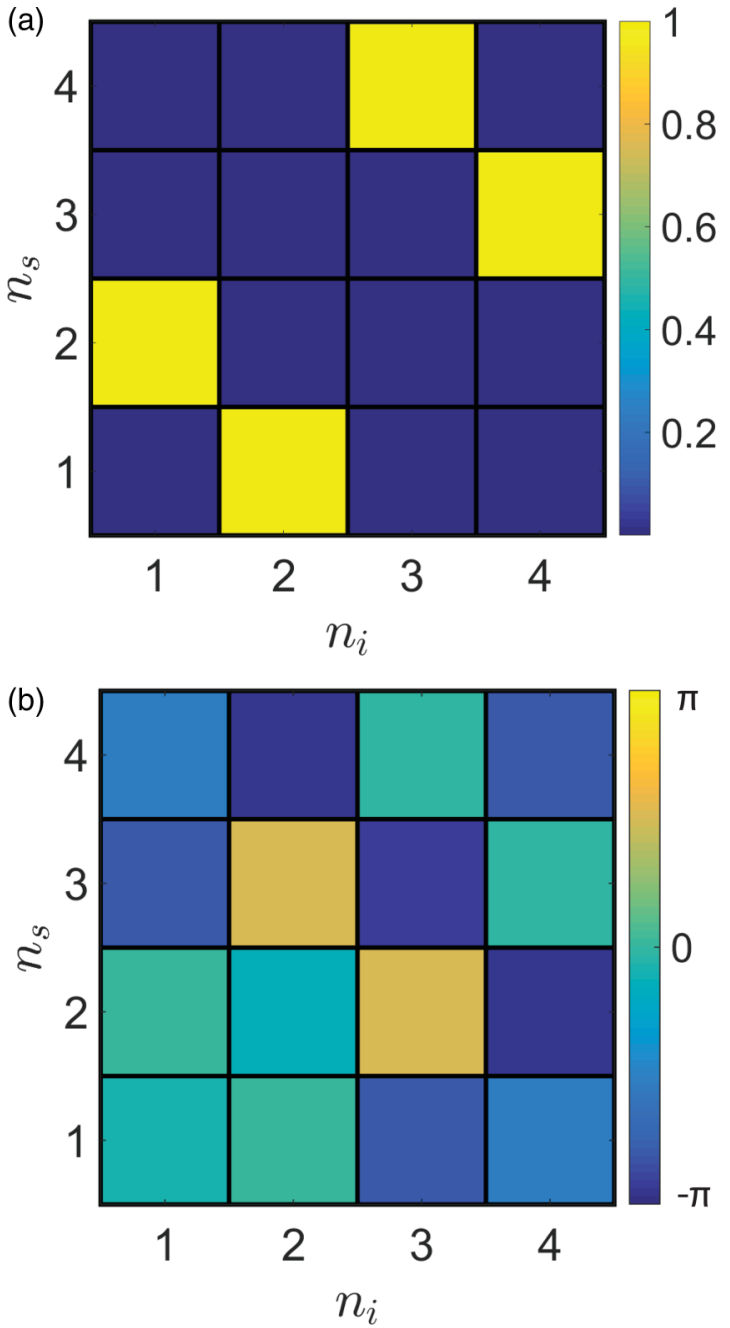

FIG. 8. (Color online) Reconstructed (a) intensity and (b) phase of the wave function $\Psi_{n_{i} n_{s}}$, using the transformation in Eq. (34) on the DFG output given in Fig. 7.

This is proportional to the expression for the down-converted wave function in Eq. (27). The same transformation can be applied to the real-space version of the DFG output

$$
E_{n_{i} k_{s^{\prime}}}^{(\text {recon })}=e^{\left(i \beta_{s^{\prime}} L\right)} \sum_{n_{s^{\prime}}=1}^{N} \sin \left(\frac{\pi n_{s^{\prime}} k_{s^{\prime}}}{N+1}\right) E_{n_{i} n_{s^{\prime}}}^{(\text {meas })} .
$$

Then, the real-space SPDC wave function can be recovered by transforming back from $k_{s^{\prime}}$ space to $n_{s}$ space

$$
\begin{aligned}
E_{n_{i} n_{s^{\prime}}}^{(\mathrm{recon})} & =\sum_{k_{s^{\prime}}=1}^{N} \sin \left(\frac{\pi n_{s} k_{s^{\prime}}}{N+1}\right) e^{\left(i \beta_{k_{s^{\prime}}} L\right)} \sum_{n_{s^{\prime}}=1}^{N} \sin \left(\frac{\pi n_{s^{\prime}} k_{s^{\prime}}}{N+1}\right) E_{n_{i} n_{s^{\prime}}}^{(\mathrm{DFG})} \\
& \propto \Psi_{n_{i} n_{s^{\prime}}}^{(\mathrm{SPDC})}
\end{aligned}
$$

This transformation is implemented in Fig. 8 to recover the quantum state produced by pumping the first waveguide of the proposed device. It would be straightforward to generalize this procedure to the case of inhomogeneous refractive indices considered in Sec. IV, so fast classical characterization could also be applied to arrays with varied refractive indices.

\section{Characterization procedure}

We have shown that a complete set of intensity and phase measurements of the DFG output of a device can be used to fully reconstruct the SPDC wave function. This will allow for quicker characterization of large numbers of devices. However, this procedure will require measurements of phase difference between light in adjacent waveguides which will be much more difficult than intensity measurements. Hence, a good first step for characterization would be to measure the intensity in each waveguide produced by DFG and compare this with the intensity predicted by Eq. (29) using the target fabrication parameters. Then, if the intensity measurements are in reasonable agreement with the target values, phase measurements could be taken to reconstruct the complete SPDC wave function. If the reconstructed wave function matches that target SPDC wave function, then finally quantum correlation measurements of down-converted photons can be used to confirm the quantum properties of the device.

This three-tiered characterization procedure will allow the majority of defective devices to be quickly identified and discarded using classical measurement techniques. Hence, the photonic chips passing such classical quality control will be suitable for operation in the quantum regime.

\section{FABRICATION TOLERANCES}

The fabrication of a nonlinear waveguide array can be thought of as involving two steps. First, the coupled modes of the waveguide array must be created by engineering the linear properties of the device. Then, the nonlinear poling pattern is imprinted on the array, allowing the waveguide modes to be driven via SPDC. Both these steps will introduce some error into the device, so the output states will deviate from the target Bell states somewhat. In this section, we analyze the effect of these errors and propose ways to compensate for the errors. We find that errors in the structural properties of the array could be mitigated by altering the poling pattern in the array and we also show how to make the poling pattern itself more robust to fabrication errors.

First, we consider errors in the fabrication of the coupled waveguides that make up the waveguide array. These errors will typically be structural errors, such as errors in the interwaveguide coupling rates, errors in the propagation constants of waveguides, or linear photon losses. These errors will all perturb the form of the supermodes of the waveguide array, resulting in errors in the output wave function. For example, we plot the fidelity of the output states in the presence of errors in the interwaveguide coupling rates in Fig. 9. This shows the fidelity decreases when the coupling rate is varied by only about $5 \%$. Therefore, it could be challenging to fabricate a waveguide array with appropriate coupling rates to realize high-fidelity quantum states from this device.

Depending on the exact fabrication procedure used to create the device, the errors mentioned above could be mitigated by using the nonlinear poling pattern of the array to compensate. This would require the waveguides to be fabricated before the poling structure is applied to the nonlinear material, as in Ref. [25]. This way, errors in the waveguide array can be characterized before the poling pattern is created. Structural 
(a)

(b)
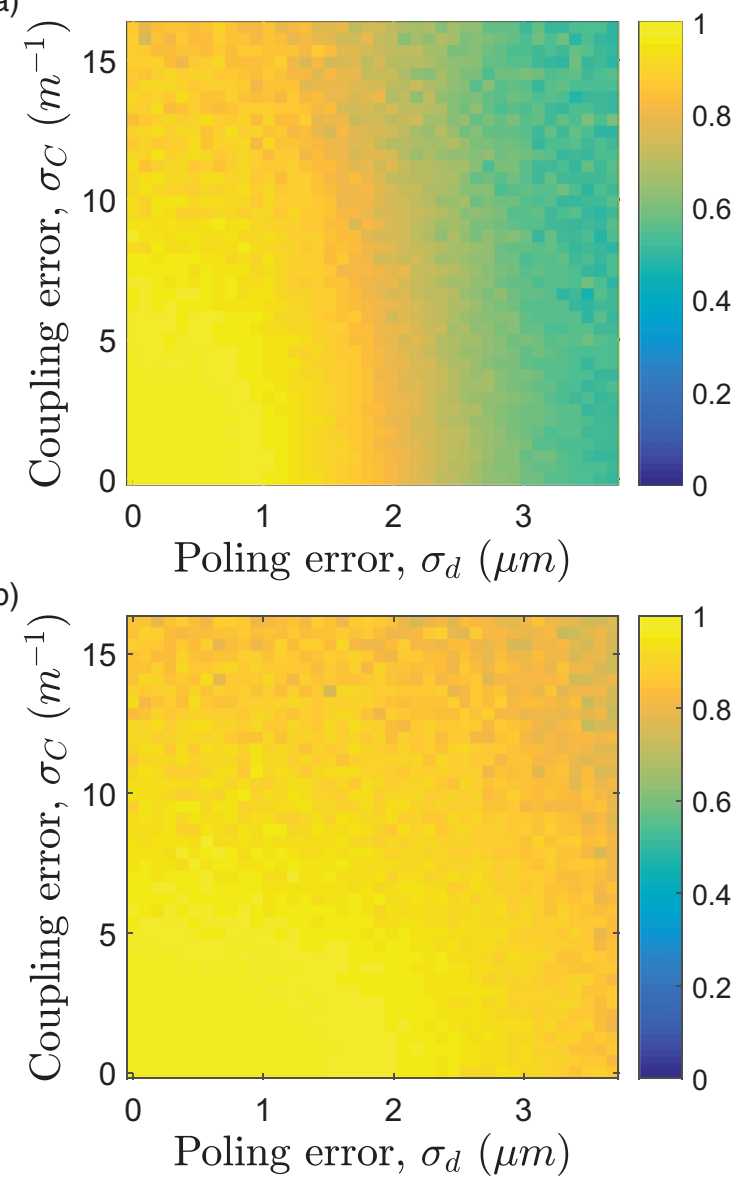

FIG. 9. (Color online) Plot of the fidelity between a target Bell state and the realized state in the presence of Gaussian errors in the coupling rates between waveguides (standard deviation $\sigma_{C}$ ) and in the location of the domain boundaries of the poling structure (standard deviation $\sigma_{d}$ ). (a) A domain poling structure with period equal to the coherence length, (b) poling period equal to twice the coherence length.

errors would perturb the supermodes of the WGA, thus the poling pattern must be adjusted to drive this new set of eigenmodes to the target output state. The mathematical method used to design the device can be adjusted to use the new set of modes rather than the typical modes considered earlier in Eq. (7). We denote the new eigenmodes by $v^{(k)}$ and the corresponding changes in propagation constant by $\delta \beta_{k_{s}}$. Adjusting Eq. (7) to account for the new modes gives

$$
\begin{aligned}
f_{k_{s}, k_{i}}(L)= & e^{i \beta_{k_{s} k_{i}} L} \sum_{n_{p}=1}^{N} A_{n_{p}} v_{n_{p}}^{\left(k_{s}\right)} v_{n_{p}}^{\left(k_{i}\right)} \\
& \times \sum_{j=1}^{M} D_{n_{p}}\left(z_{j}\right) \int_{z_{j}}^{z_{j+1}} e^{-i\left(\beta_{k_{s} k_{i}}+\delta \beta_{k_{s}}+\delta \beta_{k_{i}}\right) z} d z .
\end{aligned}
$$

Now, this equation can be inverted to solve for the aggregate nonlinearities $D_{n_{p}}\left(z_{j}\right)$, thus giving the poling pattern required to produce the target wave function $f_{k_{s}, k_{i}}(L)$ in the presence of structural errors. This applies even for errors to the propagation constants with an imaginary component, corresponding to linear losses [26]. Therefore, linear errors that can be predicted or determined by measurement could be eliminated by incorporating their effect on the system into the design of poling pattern.

Another important source of error will come from the poling structure itself, so we also consider the effect of errors in the poling pattern on the fidelity of the output wave function. We assume that errors in the poling pattern take the form of random shifts in the position of each domain wall. To model this, we add Gaussian noise to the poling structure, perturbing each domain wall by a random amount, with standard deviation denoted $\sigma_{d}(\mu \mathrm{m})$. The relationship between the standard deviation of the poling pattern $\sigma_{d}$ and the resulting fidelity of the output state are shown in Fig. 9(a). We see that high-fidelity operation can be achieved, despite Gaussian errors in the domain pattern of around $500 \mathrm{~nm}$.

Now, we consider how to make the poling pattern more robust to errors in the position of domain walls. First, it is important to realize that there are many different poling patterns that will produce the same output wave function. The important quantity is the aggregate nonlinearity of the poling pattern; this was defined earlier in Eq. (5) as

$$
D_{n_{p}}(z)=\frac{1}{\Lambda} \int_{0}^{\Lambda} e^{i \Delta \beta^{(0)} \tau} d_{n_{p}}(\tau) d \tau
$$

and represents the average amount of "down-converted wave function" produced over one coherence length $\Lambda=2 \pi / \Delta \beta^{(0)}$. This can be generalized to allow longer poling periods by integrating over an integer number of coherence lengths. The aggregate nonlinearity for a poling pattern with period $Q \Lambda$ and arbitrary duty cycle is

$$
D_{n_{p}}(z)=\frac{2}{\pi Q} \exp \left(\frac{i \pi Q l}{\Lambda}\right) \sin \left(\frac{\pi Q l}{\Lambda}\right) .
$$

By increasing the poling period to an integer multiple of the phase-matching period, there will be an increase in sizes of ferroelectric domains required in the poling structure, therefore the structure will be easier to fabricate, and furthermore errors in the domain boundary positions will have less detrimental effects. The cost of this increased ease of fabrication will be a decrease in the efficiency of the SPDC process. A plot of the fidelity of the device using a poling pattern with period equal to twice the coherence length is shown in Fig. 9(b). This shows that the device can produce a target state with high fidelity despite the position of every domain boundary in the poling structure having a standard deviation of over $1 \mu \mathrm{m}$.

Finally, increasing the poling period solves another problem: it prevents the need for arbitrarily small domain sizes. This can be seen in Eq. (37), when the poling period is twice the coherence length (so $Q=2$ ) the aggregate nonlinearity will vary from 0 to its maximum value as the duty cycle is varied from $50 \%(l / \Lambda=0.5)$ to $25 \%(l / \Lambda=0.25)$. Thus, the whole range of aggregate nonlinearities can be realized with a minimum domain size of $0.25 \Lambda$. For a typical coherence length of $18.4 \mu \mathrm{m}$, with poling period at the second harmonic $(\Lambda=32.8 \mu \mathrm{m})$, this would give a minimum domain size of $9.2 \mu \mathrm{m}$. This domain size is well within the current limits of domain poling techniques. 
To summarize, there are a number of methods that can be used to make the device more robust to errors. Linear errors can be characterized, and then mitigated by appropriate design of the poling structure. Furthermore, the accuracy of the poling structure can be increased by using longer poling periods that are integer multiples of the phase-matching length, effectively gaining accuracy at the expense of the efficiency of the down-conversion process. Finally, using a poling structure with poling period equal to twice the phase-matching period will only require a minimum domain size of approximately $9 \mu \mathrm{m}$. This is well within the limits of current technologies, therefore, the design will be possible to realize with current fabrication techniques.

\section{CONCLUSIONS}

In conclusion, we have shown how to create all-opticallyreconfigurable linear combinations of the set of two-photon Bell states in an integrated device. The device consists of four $\chi^{(2)}$ nonlinear coupled waveguides with special poling patterns in each waveguide. Since linear combinations of the Bell states span the set of all two-qubit states, the device can produce and switch between all two-qubit states simply by varying classical driving lasers. Moreover, the $\chi^{(2)}$ poling technique developed here can be applied to enable the production of any set of biphoton states, not just the four Bell states demonstrated in this work. This opens the door for the design of a variety of all-optically-reconfigurable entangled photon sources, with output quantum spaces tailored to specific technological applications. We have also shown that the device is robust to a number of sources of error, and could be fabricated with existing techniques. Finally, we demonstrate a procedure to quickly characterize the performance of such a device using only classical optics.

\section{ACKNOWLEDGMENT}

This work was supported by the Australian Research Council, including Discovery Project No. DP130100135 and Future Fellowship No. FT100100160.
[1] J. S. Bell, Physics 1, 195 (1964).

[2] P. Kok, W. J. Munro, K. Nemoto, T. C. Ralph, J. P. Dowling, and G. J. Milburn, Rev. Mod. Phys. 79, 135 (2007).

[3] N. Gisin, G. G. Ribordy, W. Tittel, and H. Zbinden, Rev. Mod. Phys. 74, 145 (2002).

[4] V. Giovannetti, S. Lloyd, and L. Maccone, Science 306, 1330 (2004).

[5] X. Q. Yu, P. Xu, Z. D. Xie, J. F. Wang, H. Y. Leng, J. S. Zhao, S. N. Zhu, and N. B. Ming, Phys. Rev. Lett. 101, 233601 (2008).

[6] H. Y. Leng, X. Q. Yu, Y. X. Gong, P. Xu, Z. D. Xie, H. Jin, C. Zhang, and S. N. Zhu, Nat. Commun. 2, 429 (2011).

[7] M. J. Collins, C. Xiong, I. H. Rey, T. D. Vo, J. He, S. Shahnia, C. Reardon, T. F. Krauss, M. J. Steel, A. S. Clark, and B. J. Eggleton, Nat. Commun. 4, 2582 (2013).

[8] H. Jin, F. M. Liu, P. Xu, J. L. Xia, M. L. Zhong, Y. Yuan, J. W. Zhou, Y. X. Gong, W. Wang, and S. N. Zhu, Phys. Rev. Lett. 113, 103601 (2014).

[9] N. K. Langford, S. Ramelow, R. Prevedel, W. J. Munro, G. J. Milburn, and A. Zeilinger, Nature (London) 478, 360 (2011).

[10] A. Politi, M. J. Cryan, J. G. Rarity, S. Y. Yu, and J. L. O’Brien, Science 320, 646 (2008).

[11] A. Orieux, A. Eckstein, A. Lemaitre, P. Filloux, I. Favero, G. Leo, T. Coudreau, A. Keller, P. Milman, and S. Ducci, Phys. Rev. Lett. 110, 160502 (2013).

[12] P. J. Shadbolt, M. R. Verde, A. Peruzzo, A. Politi, A. Laing, M. Lobino, J. C. F. Matthews, M. G. Thompson, and J. L. O'Brien, Nat. Photonics 6, 45 (2012).

[13] J. W. Silverstone, D. Bonneau, K. Ohira, N. Suzuki, H. Yoshida, N. Iizuka, M. Ezaki, C. M. Natarajan, M. G. Tanner, R. H. Hadfield, V. Zwiller, G. D. Marshall, J. G. Rarity, J. L. O'Brien, and M. G. Thompson, Nat. Photonics 8, 104 (2014).
[14] A. S. Solntsev, A. A. Sukhorukov, D. N. Neshev, and Y. S. Kivshar, Phys. Rev. Lett. 108, 023601 (2012).

[15] A. S. Solntsev, F. Setzpfandt, A. S. Clark, C. W. Wu, M. J. Collins, C. L. Xiong, A. Schreiber, F. Katzschmann, F. Eilenberger, R. Schiek, W. Sohler, A. Mitchell, C. Silberhorn, B. J. Eggleton, T. Pertsch, A. A. Sukhorukov, D. N. Neshev, and Y. S. Kivshar, Phys. Rev. X 4, 031007 (2014).

[16] C. S. Hamilton, R. Kruse, L. Sansoni, C. Silberhorn, and I. Jex, Phys. Rev. Lett. 113, 083602 (2014).

[17] A. Peruzzo, M. Lobino, J. C. F. Matthews, N. Matsuda, A. Politi, K. Poulios, X. Q. Zhou, Y. Lahini, N. Ismail, K. Worhoff, Y. Bromberg, Y. Silberberg, M. G. Thompson, and J. L. O'Brien, Science 329, 1500 (2010).

[18] M. Grafe, A. S. Solntsev, R. Keil, A. A. Sukhorukov, M. Heinrich, A. Tunnermann, S. Nolte, A. Szameit, and Y. S. Kivshar, Sci. Rep. 2, 562 (2012).

[19] M. M. Fejer, G. A. Magel, D. H. Jundt, and R. L. Byer, IEEE J. Quantum Electron. 28, 2631 (1992).

[20] Y. Q. Qin, C. Zhang, Y. Y. Zhu, X. P. Hu, and G. Zhao, Phys. Rev. Lett. 100, 063902 (2008).

[21] J. P. Torres, A. Alexandrescu, S. Carrasco, and L. Torner, Opt. Lett. 29, 376 (2004).

[22] J. Webjorn, S. Siala, D. W. Nam, R. G. Waarts, and R. J. Lang, IEEE J. Quantum Electron. 33, 1673 (1997).

[23] C. Y. J. Ying, A. C. Muir, C. E. Valdivia, H. Steigerwald, C. L. Sones, R. W. Eason, E. Soergel, and S. Mailis, Laser Photon. Rev. 6, 526 (2012).

[24] M. Liscidini and J. E. Sipe, Phys. Rev. Lett. 111, 193602 (2013).

[25] D. Janner, D. Tulli, F. Lucchi, P. Vergani, S. Giurgola, and V. Pruneri, Appl. Phys. A 91, 319 (2008).

[26] D. A. Antonosyan, A. S. Solntsev, and A. A. Sukhorukov, Phys. Rev. A 90, 043845 (2014). 